\title{
SEMICONJUGACIES BETWEEN RELATIVELY HYPERBOLIC BOUNDARIES
}

\author{
SHUBHABRATA DAS AND MAHAN MJ
}

\begin{abstract}
We prove the existence of Cannon-Thurston maps for Kleinian groups corresponding to pared manifolds whose boundary is incompressible away from cusps. We also describe the structure of these maps in terms of ending laminations.
\end{abstract}

\section{Contents}

1. Introduction

2. Background

2.1. Relative Hyperbolicity and Quasiconvexity

2.2. Cannon-Thurston Maps

2.3. Pared Manifolds

3. Reduction Theorem and Kleinian Groups

3.1. The Main Theorem

3.2. Kleinian Groups with no Accidental Parabolics 13

3.3. Accidental Parabolics 14

References

\section{INTRODUCTION}

The aim of this paper is threefold:

1) To extend the main Theorems of $\mathrm{Mj} 14 \mathrm{a}, \mathrm{Mj}$, $\mathrm{Mb}$ (which prove the existence and structure of Cannon-Thurston maps for surface groups without accidental parabolics) to Kleinian groups corresponding to pared manifolds whose boundary is incompressible away from cusps. 1 This is the content of Theorem 3.8 .

2) To give a considerably shorter and more streamlined proof of the main step of Mj09. This is the content of Theorem 3.4.

3) To generalize a reduction Theorem of Klarreich Kla99 to the context of relative hyperbolicity. This is the content of Theorem 3.1 .

The main tool, Theorem 3.1, is a 'reduction Theorem' ((3) above) which allows us to deduce the existence and structure of Cannon-Thurston maps for the inclusion of one relatively hyperbolic metric space into another, once we know the existence

\footnotetext{
Research of first author partially supported by a Department of Science and Technology Research grant. The second author is partly supported by a CSIR Junior Research Fellowship. This paper is part of SD's PhD thesis written under the supervision of MM. .

${ }^{1} \mathrm{~A}$ considerably more elaborate and somewhat clumsier proof had been sketched in an earlier version of $\mathrm{Mj} 14 \mathrm{a}$. This proof has been excised from the present version of Mj14a.
} 
and structure of Cannon-Thurston maps for inclusions of certain relatively quasiconvex subspaces into ends. The exact statement of Theorem 3.1 is somewhat technical. Suffice to say, this is the appropriate relative hyperbolic generalization of inclusions of geometrically finite hyperbolic 3-manifolds $M_{g f}$ into degenerate hyperbolic 3-manifolds $N^{h}$ such that

a) the inclusion of a boundary component $S_{g f}$ of $M_{g f}$ into the end $E^{h}$ of $N^{h}$ it bounds is a homotopy equivalence.

b) Each $S_{g f}$ is incompressible in $M_{g f}$.

We give the main application below.

Theorem 3.8: Suppose that $N^{h} \in H(M, P)$ is a hyperbolic structure on a pared manifold $(M, P)$ with incompressible boundary $\partial_{0} M$. Suppose further that $N^{h}$ is not doubly degenerate. Let $M_{g f}$ denotes a geometrically finite hyperbolic structure adapted to $(M, P)$. Then the map $i: \widetilde{M_{g f}} \rightarrow \widetilde{N^{h}}$ extends continuously to the boundary $\hat{i}: \widehat{M_{g f}} \rightarrow \widehat{N^{h}}$.

Let $\partial i: \partial \widetilde{M_{g f}} \rightarrow \partial \widetilde{N^{h}}$ be the resulting Cannon-Thurston map extending $i$ : $\widetilde{M_{g f}} \rightarrow \widetilde{N^{h}}$. Then $\partial i(a)=\partial i(b)$ for $a \neq b$ if and only if $(a, b) \in \mathcal{R}$, where $\mathcal{R}$ is the smallest closed equivalence relation containing the equivalence relations generated by lifts of the ending laminations to $\widetilde{M_{g f}}$.

The last statement is informally abbreviated by saying that the Cannon-Thurston map identifies precisely the end-points of leaves of the ending laminations. (Note that we have to pass to the transitive closure to get a precise statement.) It is curious that the doubly degenerate case (dealt with in [Mj14a, $\mathrm{Mj} 14 \mathrm{~b}]$ ) is the single exceptional case not amenable to the techniques of this paper.

The last step of the programme of proving the existence of Cannon-Thurston maps for arbitrary finitely generated Kleinian groups and describing their structure is dealt with in $\mathrm{Mj} 10$.

Acknowledgments: The authors would like to thank the referee for a careful reading and detailed and helpful comments and also for pointing out a gap in an earlier draft.

\section{BACKGROUND}

2.1. Relative Hyperbolicity and Quasiconvexity. Let $(X, d)$ be a path metric space. A collection of closed subsets $\mathcal{H}=\left\{H_{\alpha}\right\}$ of $X$ will be said to be uniformly separated if there exists $D>0$ such that $d\left(H_{1}, H_{2}\right) \geq D$ for all distinct $H_{1}, H_{2} \in$ $\mathcal{H}$.

Definition 2.1. (Farb Far98) The electric space (or coned-off space) $\mathcal{E}(X, \mathcal{H})$ corresponding to the pair $(X, \mathcal{H})$ is a metric space which consists of $X$ and a collection of vertices $v_{\alpha}$ (one for each $H_{\alpha} \in \mathcal{H}$ ) such that each point of $H_{\alpha}$ is joined to (coned off at) $v_{\alpha}$ by an edge of length $\frac{1}{2}$. The sets $H_{\alpha}$ shall be referred to as horosphere-like sets and the vertices $v_{\alpha}$ as cone-points.

$X$ is said to be weakly hyperbolic relative to the collection $\mathcal{H}$ if $\mathcal{E}(X, \mathcal{H})$ is a hyperbolic metric space.

Definition 2.2. A path $\gamma$ is said to be an electric geodesic (resp. electric $K$ quasigeodesic) if it is a geodesic (resp. $K$-quasigeodesic) in $\mathcal{E}(X, \mathcal{H})$. 
$\gamma$ is said to be an electric $K$-quasigeodesic in (the electric space) $\mathcal{E}(X, \mathcal{H})$ without backtracking if $\gamma$ is an electric $K$-quasigeodesic in $\mathcal{E}(X, \mathcal{H})$ and $\gamma$ does not return to any horosphere-like set $H_{\alpha}$ after leaving it.

Let $i: X \rightarrow \mathcal{E}(X, \mathcal{H})$ denotes the natural inclusion of spaces. Then for a path $\gamma \subset X$, the path $i(\gamma)$ lies in $\mathcal{E}(X, \mathcal{H})$. Replacing maximal subsegments $[a, b]$ of $i(\gamma)$ lying in a particular $H_{\alpha}$ by a path that goes from $a$ to $v_{\alpha}$ and then from $v_{\alpha}$ to $b$, and repeating this for every $H_{\alpha}$ that $i(\gamma)$ meets we obtain a new path $\hat{\gamma}$. If $\hat{\gamma}$ is an electric geodesic (resp. $P$-quasigeodesic), $\gamma$ is called a relative geodesic (resp. relative $P$-quasigeodesic). Paths (resp. geodesics or quasigeodesics) in $X$ shall be referred to as ambient paths (resp. geodesics or quasigeodesics). As above, an ambient path is said to be without backtracking if it does not return to any horosphere-like set $H_{\alpha}$ after leaving it. We shall usually be concerned with the case that $\gamma$ is an ambient geodesic/quasigeodesic without backtracking.

Definition 2.3. Relative $P$-quasigeodesics in $(X, \mathcal{H})$ are said to satisfy bounded region penetration if there exists $B=B(P)$ so that for any two relative $P$ quasigeodesics without backtracking $\beta, \gamma$, joining $x, y$ we have

Similar Intersection Patterns 1: if precisely one of $\beta, \gamma$ meets a horosphere-like set $H_{\alpha}$, then the length of this path (measured in the intrinsic path-metric on $H_{\alpha}$ ) from the first (entry) point to the last (exit) point (of the relevant path) is at most $B$.

Similar Intersection Patterns 2: if both $\beta, \gamma$ meet some $H_{\alpha}$ then the distance (measured in the intrinsic path-metric on $H_{\alpha}$ ) from the entry point of $\beta$ to that of $\gamma$ is at most B; similarly for exit points.

Replacing ' $P$-quasigeodesic' by 'geodesic' in the above definition, we obtain the notion of relative geodesics in $(X, \mathcal{H})$ satisfying bounded region penetration.

Families of paths which enjoy the above properties shall be said to have similar intersection patterns with horospheres.

Definition 2.4. (Farb Far98 ) $X$ is said to be hyperbolic relative to the uniformly separated collection $\mathcal{H}$ if

1) $X$ is weakly hyperbolic relative to $\mathcal{H}$.

2) For all $P \geq 1$, relative $P$-quasigeodesics without backtracking satisfy the bounded penetration property.

Elements of $\mathcal{H}$ will be referred to as horosphere-like sets.

\section{Gromov's definition of relative hyperbolicity Gro85 :}

Definition 2.5. (Gromov) For any geodesic metric space $(H, d)$, the hyperbolic cone (analog of a horoball) $H^{h}$ is the metric space $H \times[0, \infty)=H^{h}$ equipped with the path metric $d_{h}$ defined by

1) $d_{h, t}((x, t),(y, t))=2^{-t} d_{H}(x, y)$, where $d_{h, t}$ is the induced path metric on $H \times\{t\}$. Paths joining $(x, t),(y, t)$ and lying on $H \times\{t\}$ are called horizontal paths.

2) $d_{h}((x, t),(x, s))=|t-s|$ for all $x \in H$ and for all $t, s \in[0, \infty)$, and the corresponding paths are called vertical paths.

3) For all $x, y \in H^{h}, d_{h}(x, y)$ is the path metric induced by the collection of horizontal and vertical paths. 
Definition 2.6. Let $X$ be a geodesic metric space and $\mathcal{H}$ be a collection of mutually disjoint uniformly separated subsets of $X$. The space $X$ is said to be hyperbolic relative to $\mathcal{H}$ in the sense of Gromov, if the quotient space $\mathcal{G}(X, \mathcal{H})$, obtained by attaching the hyperbolic cones $H^{h}$ to $H \in \mathcal{H}$ by identifying $(z, 0)$ with $z$ for all $H \in$ $\mathcal{H}$ and $z \in H$, is a complete hyperbolic metric space. The collection $\left\{H^{h}: H \in \mathcal{H}\right\}$ is denoted as $\mathcal{H}^{h}$. The induced path metric is denoted as $d_{h}$.

We shall refer to $\mathcal{G}(X, \mathcal{H})$ as the Gromov cone for the pair $(X, \mathcal{H})$.

Theorem 2.7. (Bowditch Bow12) The following are equivalent:

1) $X$ is hyperbolic relative to the collection $\mathcal{H}$ of uniformly separated subsets of $X$.

2) $X$ is hyperbolic relative to the collection $\mathcal{H}$ of uniformly separated subsets of $X$ in the sense of Gromov.

3) $\mathcal{G}(X, \mathcal{H})$ is hyperbolic relative to the collection $\mathcal{H}^{h}$.

Definition 2.8. Let $X$ be hyperbolic relative to the collection $\mathcal{H}$. We call a set $W \subset X$ relatively $K$-quasiconvex if

1) $W$ is hyperbolic relative to the collection $\mathcal{W}=\{W \cap H: H \in \mathcal{H}\}$

2) $\mathcal{E}(W, \mathcal{W})$ is $K$-quasiconvex in $\mathcal{E}(X, \mathcal{H})$.

$W \subset X$ is relatively quasiconvex if it is relatively $K$-quasiconvex for some $K$.

Ends:

Let $Y$ be hyperbolic rel. $\mathcal{H}$. Now let $\mathcal{B}=\left\{B_{\alpha}\right\}, \alpha \in \Lambda$, for some indexing set $\Lambda$, be a collection of uniformly relatively quasiconvex sets inside $Y$. Here each $B_{\alpha}$ is relatively quasiconvex with respect to the collection $\left\{B_{\alpha \beta}\right\}$, given by $B_{\alpha \beta}=B_{\alpha} \cap H_{\beta}$. We also assume that the sets $H_{\beta}$ are uniformly $D$-separated.

Definition 2.9. Let $Y$ be hyperbolic relative to the collection $\mathcal{H}$ and $X$ be strongly hyperbolic with respect to a collection $\mathcal{J}$. A map $i: Y \rightarrow X$ is said to be strictly type-preserving if:

1) For every $H \in \mathcal{H}, i(H) \subset J_{H}$ for some $J_{H} \in \mathcal{J}$.

2) For every $J \in \mathcal{J}, i^{-1}(J)=\emptyset$ or $i^{-1}(J)=H_{J}$ for some $H_{J} \in \mathcal{H}$

A map of path-metric spaces is a length-isometry if it preserves lengths of paths.

Definition 2.10. A strictly type-preserving length-isometric inclusion $i: Y \hookrightarrow X$ of relatively hyperbolic metric spaces is said to be an ends-inclusion if

1) There exist collections $\mathcal{J}=\left\{J_{\beta}\right\}, \mathcal{H}=\left\{H_{\beta}\right\}$ such that $X$ is hyperbolic rel. $\mathcal{J}$ and $Y$ is hyperbolic rel. $\mathcal{H}$ (note that $\beta$ ranges in the same indexing set).

2) There exists a collection $\mathcal{B}=\left\{B_{\alpha}\right\}, \alpha \in \Lambda$, of relatively quasiconvex subsets of $Y$. Each $B_{\alpha}$ is relatively quasiconvex with respect to the collection $\left\{B_{\alpha \beta}\right\}$ given by $B_{\alpha \beta}=B_{\alpha} \cap H_{\beta}$.

3) There exists a collection $\mathcal{F}=\left\{F_{\alpha} \subset X\right\}, \alpha \in \Lambda$, of relatively quasiconvex subsets of $X$ (thought of as ends of $X$ ), such that $B_{\alpha}=F_{\alpha} \cap Y, \forall \alpha$ and $X=Y \cup\left\{\bigcup_{\alpha} F_{\alpha}\right\}$. We also have the inclusion maps $i_{\alpha}: B_{\alpha} \rightarrow F_{\alpha}$.

4) Each $F_{\alpha}$ is strongly hyperbolic relative to the collection $\left\{F_{\alpha \beta}=F_{\alpha} \cap J_{\beta}\right\}$.

5) If $\mathcal{H}_{0}$ is the subcollection of elements $H_{\gamma} \in \mathcal{H}$ such that $H_{\gamma} \cap F_{\alpha}=\emptyset$ for all $F_{\alpha}$, then $\mathcal{J}=\mathcal{H}_{0} \cup \bigcup_{\alpha, \beta}\left\{F_{\alpha \beta}\right\}$.

We let $\mathcal{H}_{1}=\mathcal{H} \backslash \mathcal{H}_{0}$.

Remark 2.11. It might be useful here to keep the motivating example of a pared hyperbolic 3-manifold $N$ with incompressible boundary (cf. Definition 2.22 below) 
in mind. We give an informal sketch of the setup to fix notions. In this situation, there exists a geometrically finite manifold $M$ and an embedding $i: M \rightarrow N$ such that $N \backslash M$ consists of finitely many products of the form $S \times[0, \infty)$ for $S$ a finite area hyperbolic surface. Then $X$ (resp. $Y$ ) in Definition 2.10 corresponds to the universal cover of $N$ (resp. $M$ ). The lifts of the $S \times\{0\}$ 's correspond to $\left\{B_{\alpha}\right\}$. The lifts of the cusps of the $S \times[0, \infty)$ 's correspond to $\left\{F_{\alpha \beta}\right\}$. There might be cusps in $M$ which have no curves parallel to the cusps of the $S \times\{0\}$ 's. Lifts of such cusps correspond to $\mathcal{H}_{0}$. Finally, the lifts of the cusps of the $S \times\{0\}$ 's correspond to $\mathcal{H}_{1}$.

$M$ is often referred to as the relative Scott core of $N$.

Remark 2.12. Note that the ends-inclusion $i: Y \hookrightarrow X$ induces an isometric embedding $\hat{i}: \mathcal{E}(Y, \mathcal{B}) \rightarrow \mathcal{E}(X, \mathcal{F})$. Further, every point of $\mathcal{E}(X, \mathcal{F})$ is within bounded distance (in fact distance $\frac{1}{2}$ ) of the image of $\mathcal{E}(Y, \mathcal{B})$. The points of $\mathcal{E}(X, \mathcal{F})$ not in the image of $\mathcal{E}(Y, \mathcal{B})$ correspond precisely to points of $F_{\alpha} \backslash i_{\alpha}\left(B_{\alpha}\right)$ for some $\alpha$. It follows that for any electric geodesic (resp. $P$ - quasigeodesic) $\sigma$ in $\mathcal{E}(Y, \mathcal{B})$, $\hat{i}(\sigma)$ is an electric geodesic (resp. $P$ - quasigeodesic) in $\mathcal{E}(X, \mathcal{F})$.

Lemma 2.13. Bow12 Let $X$ be a hyperbolic metric space and let $\mathcal{B}$ be a collection of uniformly separated uniformly quasiconvex sets. Then $X$ is weakly hyperbolic relative to the collection $\mathcal{B}$.

Let $X$ be a $\delta$-hyperbolic metric space, and $\mathcal{B}$ a family of $C$-quasiconvex, $D$ separated, collection of subsets. Then by Lemma 2.13 (see also [Far98), $X_{e l}=$ $\mathcal{E}(X, \mathcal{B})$ obtained by electrocuting the subsets in $\mathcal{B}$ is a $\Delta=\Delta(\delta, C, D)$-hyperbolic metric space. Now, let $\alpha=[a, b]$ be a hyperbolic geodesic in $X$ and $\beta$ be an electric $P$-quasigeodesic without backtracking joining $a, b$. Replace each maximal subsegment, (with end-points $p, q$, say) starting from the left of $\beta$ lying within some $H \in \mathcal{H}$ by a hyperbolic geodesic $[p, q]$. The resulting connected path $\beta_{\text {ea }}$ is called an electro-ambient path representative in $X$.

Note that $\beta_{\text {ea }}$ need not be a hyperbolic quasigeodesic. However, the proof of Proposition 4.3 of Klarreich Kla99 gives the following. (See Mj14a, Lemma 2.5] for a proof of the forward direction. The converse direction follows directly from the proof of [Kla99, Proposition 4.3].)

Lemma 2.14. Given $\delta, C, D, P$ there exists $C_{3}$ such that the following holds: Let $(X, d)$ be a $\delta$-hyperbolic metric space and $\mathcal{H}$ a family of $C$-quasiconvex, $D$ separated collection of quasiconvex subsets. Let $\left(X, d_{e}\right)$ denote the electric space obtained by electrocuting elements of $\mathcal{H}$. Then, if $\alpha, \beta_{\text {ea }}$ denote respectively a hyperbolic geodesic and an electro-ambient $P$-quasigeodesic with the same end-points, then $\alpha$ lies in a (hyperbolic) $C_{3}$ neighborhood of $\beta_{e a}$.

Conversely, given a hyperbolic geodesic $\alpha$, there exists an electro-ambient $P-$ quasigeodesic $\gamma_{e a}$ lying in a (hyperbolic) $C_{3}$ neighborhood of $\alpha$.

We shall abbreviate this as:

Hyperbolic geodesics lies hyperbolically close to electro-ambient representatives of electric geodesics joining their end-points. Conversely, given a hyperbolic geodesic there is an electro-ambient quasigeodesic lying close to it.

A word of clarification here regarding the hypotheses of Lemma 2.14 $D$ - separatedness is only a technical assumption. Given $X, \mathcal{H}$, let $X_{1}=X \bigcup_{H \in \mathcal{H}}(H \times[0,1])$, equipped with the quotient topology, where $(h, 0) \in(H \times[0,1])$ is identified with $h \in H \subset X$. Then the collection $\{H \times\{1\}: H \in \mathcal{H}\}$ is automatically 2-separated 
and the inclusion of $X$ in $Y$ is a quasi-isometry. However, the requirement that each $H$ is $C$ - quasiconvex is an essential assumption and the conclusion of Lemma 2.14 fails without this assumption. It is not sufficient to assume that $X$ is (weakly) hyperbolic relative to the collection $\mathcal{H}$. A simple counterexample is given by a doubly degenerate 3-manifold, with the 2 ends corresponding to the 2 elements of $\mathcal{H}$. We are grateful to the referee for bringing this to our notice.

2.2. Cannon-Thurston Maps. For a hyperbolic metric space $X$, the Gromov bordification will be denoted by $\bar{X}$.

Definition 2.15. Let $X$ and $Y$ be hyperbolic metric spaces and $i: Y \rightarrow X$ be an embedding. A Cannon-Thurston map $\bar{i}$ from $\bar{Y}$ to $\bar{X}$ is a continuous extension of $i$ to the Gromov bordifications $\bar{X}$ and $\bar{Y}$.

The following lemma from Mit98 gives a necessary and sufficient condition for the existence of Cannon-Thurston maps.

Lemma 2.16. Mit98 A Cannon-Thurston map $\bar{i}$ from $\bar{Y}$ to $\bar{X}$ exists for the proper embedding $i: Y \rightarrow X$ if and only if there exists a non-negative function $M(N)$ with $M(N) \rightarrow \infty$ as $N \rightarrow \infty$ such that the following holds:

Given $y_{0} \in Y$, for all geodesic segments $\lambda$ in $Y$ lying outside an $N$-ball around $y_{0} \in Y$, any geodesic segment in $X$ joining the end points of $i(\lambda)$ lies outside the $M(N)$-ball around $i\left(y_{0}\right) \in X$.

Note that due to stability of quasigeodesics, the above statement is also true if geodesics are replaced by uniform quasigeodesics.

Let $X$ and $Y$ be hyperbolic relative to the collections $\mathcal{H}_{X}$ and $\mathcal{H}_{Y}$ respectively. Let $\widehat{X}=\mathcal{E}\left(X, \mathcal{H}_{X}\right), \widehat{Y}=\mathcal{E}\left(Y, \mathcal{H}_{Y}\right)$. Let $i: Y \rightarrow X$ be a strictly type-preserving proper embedding. Then the proper embedding $i: Y \rightarrow X$ induces a proper embedding $i_{h}: \mathcal{G}\left(Y, \mathcal{H}_{Y}\right) \rightarrow \mathcal{G}\left(X, \mathcal{H}_{X}\right)$ and a map $\hat{i}: \widehat{X} \rightarrow \widehat{Y}$.

Definition 2.17. A Cannon-Thurston map is said to exist for the pair $X, Y$ of relatively hyperbolic metric spaces and a strictly type-preserving inclusion $i: Y \rightarrow X$ if a Cannon-Thurston map exists for the induced map $i_{h}: \mathcal{G}\left(Y, \mathcal{H}_{Y}\right) \rightarrow \mathcal{G}\left(X, \mathcal{H}_{X}\right)$.

In MP11] Lemma 2.16 was generalized to relatively hyperbolic metric spaces as follows.

Lemma 2.18. (MP11 Lemma 1.28) Let $Y, X$ be hyperbolic rel. $\mathcal{Y}, \mathcal{X}$ respectively. Let $Y^{h}=\mathcal{G}(Y, \mathcal{Y}), \widehat{Y}=\mathcal{E}(Y, \mathcal{Y})$ and $X^{h}=\mathcal{G}(X, \mathcal{X}), \widehat{X}=\mathcal{E}(X, \mathcal{X})$. A CannonThurston map for $i: Y \rightarrow X$ exists if and only if there exists a non-negative function $M(N)$ with $M(N) \rightarrow \infty$ as $N \rightarrow \infty$ such that the following holds:

Suppose $y_{0} \in Y$, and $\hat{\lambda}$ in $\hat{Y}$ is an electric geodesic segment starting and ending outside horospheres. If $\lambda^{b}=\hat{\lambda} \backslash \bigcup_{K \in \mathcal{Y}} K$ lies outside $B_{N}\left(y_{0}\right) \subset Y$, then for any electric quasigeodesic $\hat{\beta}$ joining the end points of $\hat{i}(\hat{\lambda})$ in $\widehat{X}, \beta^{b}=\hat{\beta} \backslash \bigcup_{H \in \mathcal{X}} H$ lies outside $B_{M(N)}\left(i\left(y_{0}\right)\right) \subset X$.

The above necessary and sufficient condition for existence of Cannon-Thurston map for relatively hyperbolic spaces can also be used as a definition of CannonThurston map for relatively hyperbolic spaces. Hence the following definition makes sense. 
Definition 2.19. A collection of proper, strictly type preserving embedding $i_{\alpha}$ : $Y_{\alpha} \rightarrow X_{\alpha}$ of relatively hyperbolic spaces is said to extend to a collection of uniform Cannon-Thurston maps if there exists $M(N) \rightarrow \infty$ as $N \rightarrow \infty$ such that the functions $M_{\alpha}(N)$ (obtained in Lemma 2.18 above) satisfy $M_{\alpha}(N) \geq M(N)$ for all $\alpha$.

We shall often abbreviate Cannon-Thurston as CT in what follows. Lemma 2.18 says that it is enough to consider only the 'bounded'-part of the electric quasigeodesic in a relatively hyperbolic space $X$ in order to prove existence of $\mathbf{C T}$ map. For ease of reference below, we make the following definition.

Definition 2.20. Let $X$ be hyperbolic rel. $\mathcal{X}$. If $\sigma$ is a path in $X$, the bounded part $\sigma^{b}$ of $\sigma$ with respect to $(X, \mathcal{X})$ is defined as $\sigma \backslash \bigcup_{H \in \mathcal{X}} H$.

If there is no ambiguity, we shall refer to the bounded part of $\sigma$ with respect to $(X, \mathcal{X})$ simply as the bounded part of $\sigma$.

We shall use the notion of electro-ambient path representatives to obtain an alternate criterion for the existence of Cannon-Thurston maps in the case of an ends-inclusion. Combining Lemma 2.18 with Lemma 2.14 we have the following.

Lemma 2.21. Let $X, Y$ be hyperbolic rel. $\mathcal{J}, \mathcal{H}$ respectively and $i: Y \rightarrow X$ be an ends-inclusion of relatively hyperbolic spaces. A Cannon-Thurston map for $i: Y \hookrightarrow$ $X$ exists if and only there exists a non-negative function $M(N)$ with $M(N) \rightarrow \infty$ as $N \rightarrow \infty$ such that the following holds.

Suppose $y \in Y$, and $\hat{\lambda}$ in $\widehat{Y}$ is an electric geodesic segment starting and ending outside horospheres, such that $\lambda^{b}=\hat{\lambda} \backslash \bigcup_{K \in \mathcal{H}} K$, the bounded part of $\hat{\lambda}$ lies outside $B_{N}(y) \subset Y$.

Then for some electric quasigeodesic $\hat{\rho}$ joining the end points of $\hat{i}(\hat{\lambda})$ in $\widehat{X}$, the bounded part $\rho_{e a}^{b}=\hat{\rho}_{e a} \backslash \cup_{H \in \mathcal{J}} H$ of the electro-ambient representative $\rho_{e a}$ (of $\hat{\rho}$ ) lies outside $B_{M(N)}(i(y)) \subset X$.

2.3. Pared Manifolds. The main examples of interest in this paper are pared 3-manifolds.

Definition 2.22. A pared manifold is a pair $(M, P)$, where $M$ is a compact irreducible 3-manifold with boundary $\delta M$ and $P \subset \delta M$ is a (possibly empty) 2dimensional submanifold with boundary (of $\delta M$ ) such that

(1) Any $\pi_{1}$-injective map of a torus or Klein bottle into $M$ is homotopic to a map into $\delta M$.

(2) The fundamental group of each component of $P$ injects into the fundamental group of $M$.

(3) The fundamental group of each component of $P$ contains an abelian subgroup with finite index.

(4) Any map $C:\left(S^{1} \times I, \delta\left(S^{1} \times I\right)\right) \rightarrow(M, P)$ such that $\pi_{1}(C)$ is injective, is homotopic rel boundary to $P$.

(5) $P$ contains every component of $\delta M$ which has an abelian subgroup of finite index.

A pared manifold $(M, P)$ is said to have incompressible boundary if each component of $\delta_{0} M=\delta M \backslash P$ is incompressible in $M$.

Further, $(M, P)$ is said to have no accidental parabolics if 
(1) It has incompressible boundary.

(2) If some curve $\sigma$ on a component of $\delta_{0} M$ is freely homotopic in $M$ to a curve $\alpha$ on a component of $P$, then $\sigma$ is homotopic to $\alpha$ in $\delta M$.

Definition 2.23. Thu86a, Thu86b A hyperbolic structure on a pared manifold $(M, P)$ is defined to be a complete hyperbolic structure on the interior of $M$ given by a discrete faithful representation $\rho: \pi_{1}(M) \rightarrow I s o m\left(\mathbb{H}^{3}\right)$ such that any homotopically nontrivial loop in $M$ represented by a parabolic is homotopic into $P$. Further, for any component $P_{i}$ of $P$, and any homotopically essential curve $\gamma$ in $\pi_{1}\left(P_{i}\right)\left(\subset \pi_{1}(M)\right), \rho(\gamma)$ is a parabolic.

The space of hyperbolic structures on $(M, P)$ is denoted by $H(M, P)$.

Let $\Gamma=\rho\left(\pi_{1}(M)\right) \subset \operatorname{Isom}\left(\mathbb{H}^{3}\right)$. A hyperbolic structure on $(M, P)$ is said to be geometrically finite (resp. infinite) if $\Gamma$ is a geometrically finite (resp. infinite) Kleinian group. Thurston's hyperbolization theorem Thu86a, Thu86b, Kap01, Ota98 ensures that $H(M, P)$ contains a geometrically finite structure $M_{g f}$. Further, the limit set of a geometrically finite $\Gamma$ is equivariantly homeomorphic to the boundary of the Gromov cone $\mathcal{G}(X, \mathcal{H})$ where $X$ is the universal cover $\widetilde{M}$ and the parabolic subgroups $\mathcal{H}$ correspond to the fundamental groups of the components of $P$. Very often, in what follows we shall not be considering all of $\mathbb{H}^{3} / \Gamma$ but rather its convex core, or equivalently, the quotient of the convex hull of the limit set of $\Gamma$ by $\Gamma$. By slight abuse of notation, we shall continue to denote the convex core of $M_{g f}$ by $M_{g f}$.

We give a slightly different but equivalent description of accidental parabolics in terms of hyperbolic structures on $(M, P)$. Recall (Definition 2.22 above) that for a pared manifold $(M, P)$, any map $C:\left(S^{1} \times I, \delta\left(S^{1} \times I\right)\right) \rightarrow(M, P)$ of an annulus such that $\pi_{1}(C)$ is injective, is homotopic rel boundary to $P$. An element $\gamma \in \Gamma$ is an accidental parabolic, if the converse is false, i.e.

a) If there exists a homotopically essential map $C:\left(S^{1} \times I, \delta\left(S^{1} \times I\right)\right) \rightarrow(M, P)$ such that $C\left(S^{1} \times\{0\}\right)$ is contained in $\delta_{0} M, C\left(S^{1} \times\{1\}\right)$ is contained in a component $P_{i}$ of $P$, but $C$ is not homotopic rel. boundary to a map $\left(S^{1} \times I, \delta\left(S^{1} \times I\right)\right) \rightarrow \delta M$. b) a geodesic representative of $\gamma$ in $M$ is freely homotopic to the core curve of the annulus $C\left(S^{1} \times I\right)$.

A component $P_{i}$ of $P$ for which such a map $C$ exists is called exceptional.

In summary an accidental parabolic is given by the core curve of a homotopically essential map $C:\left(S^{1} \times I, \delta\left(S^{1} \times I\right)\right) \rightarrow(M, P)$ of an annulus into a pared manifold $(M, P)$ such that

a) $C\left(S^{1} \times\{0\}\right)$ is contained in $\delta_{0} M(=\delta M \backslash P)$,

b) $C\left(S^{1} \times\{1\}\right)$ is contained in a component $P_{i}$ of $P$,

c) $C$ is not homotopic rel. boundary to a map $\left(S^{1} \times I, \delta\left(S^{1} \times I\right)\right) \rightarrow \delta M$.

For a hyperbolic structure $N^{h} \in H(M, P)$ adapted to $(M, P)$, an exceptional cusp is a cusp corresponding to an exceptional component $P_{i}$. Exceptional horoballs are lifts of (neighborhoods of) exceptional cusps. Boundaries of exceptional horoballs are called exceptional horospheres.

We now describe how to adjoin exceptional cusps to ends having accidental parabolics so that the resulting set can be treated on an equal footing with ends containing no accidental parabolics.

Let $E$ be an end of $N^{h}$ and $\Sigma \subset \delta M$ be its boundary. Let $\sigma_{1}, \cdots \sigma_{k} \subset \Sigma$ be all the simple closed curves on $\Sigma$ corresponding to accidental parabolics. Then each $\sigma_{i}$ is homotopic into an exceptional cusp and there is an embedded annulus $A_{i}$ with 
one boundary component $\sigma_{i}$ and the other component $\sigma_{i}^{\prime}$ in the exceptional cusp. We choose $\sigma_{i}^{\prime}$ to be geodesic in the canonical flat metric on the boundary of the exceptional cusp. Then $\sigma_{i}^{\prime}$ bounds a totally geodesic annulus $C_{i}$ contained in the exceptional cusp bounded by $\sigma_{i}^{\prime}$ and isometric to the quotient of a 2-dimensional horodisk by a cyclic parabolic group. Note that if the exceptional cusp is rank one, then $C_{i}$ equals the exceptional cusp. The union $E \bigcup_{i}\left(A_{i} \bigcup_{i} C_{i}\right)$ will be termed an augmented end.

We shall need the Thurston-Canary covering theorem [Thu80][Ch. 9] Can96] in the context of pared manifolds. The version below combines the covering theorem with the tameness theorems of Bonahon, Agol and Gabai-Calegari Bon86, Ago04, GC06.

Theorem 2.24. Thu80 Can96 Let $M=\mathbf{H}^{3} / \Gamma$ be a complete hyperbolic 3manifold. A finitely generated subgroup $\Gamma^{\prime}$ is geometrically infinite if and only if it contains a finite index subgroup of a geometrically infinite peripheral subgroup.

Another fact we shall need in this context is the following (see also [Can96]):

Lemma 2.25. Let $E$ be an augmented degenerate end for a hyperbolic structure $N^{h}$ on a pared manifold $(M, P)$ with incompressible boundary. Let $\widetilde{E}$ be a lift of $E$ to $\widetilde{M}$, equipped with this hyperbolic structure. Then $\widetilde{E}$ is not relatively quasiconvex in $\widetilde{N^{h}}$ if and only if there is a component $F$ of $\delta_{0} M$ such that

(1) $F$ bounds a degenerate end other than $E$, and

(2) $F$ is homotopic into E.

Proof. The proof is essentially a rerun of some of the arguments appearing in Bon86. Suppose $\widetilde{E}$ is not relatively quasiconvex in $\widetilde{N^{h}}$. Then there exists a sequence of closed curves $\sigma_{i}$ on $\partial E$ whose geodesic realizations $\gamma_{i}$ in $N^{h}$ satisfy $d\left(\gamma_{i}, E\right) \rightarrow \infty$ as $i \rightarrow \infty$ (Section 2.2 of Bon86] proves the existence of closed curves satisfying the above property). Then (cf. Proposition 5.1 of [Bon86]) a subsequence of the $\sigma_{i}$ 's converges to an ending lamination $\Lambda$ on $\partial E$. If $\Lambda_{E}$ is the ending lamination for the end $E$, then $\Lambda$ is different from $\Lambda_{E}$.

If the support of $\Lambda$ is all of $\partial E$ and $\Lambda$ contains no simple closed curve, then $N^{h}$ is doubly degenerate. Else any simple closed curve in $\Lambda$ gives rise to an accidental parabolic. Let $F$ be a connected subsurface of $\partial E$ supporting an ending lamination contained in $\Lambda$. Then $F$ satisfies the conclusions of the Lemma.

\section{Reduction Theorem and Kleinian Groups}

3.1. The Main Theorem. Before stating the main Reduction Theorem3.1below, we briefly sketch the proof idea in the special case of hyperbolic 3-manifolds $N$ with incompressible core $M$ and no parabolics. For concreteness, suppose that $N$ has one end and that the end $E=N \backslash M$ is homeomorphic to $S \times[0, \infty)$ for a compact hyperbolic surface $S$. Theorem 3.1 says in this case that if the inclusion of $\widetilde{S}$ into $\widetilde{E}$ has a CT map, then so does the inclusion of $\widetilde{M}$ into $\widetilde{N}$. The proof idea is as follows:

Let $\mathcal{E}$ denote the collection of lifts of the end $E$ to $\widetilde{N}$ and let $\mathcal{S}$ denote the collection of lifts of $S$ to $\widetilde{M}$. Then by Lemma 2.25, each lift $E_{\alpha} \in \mathcal{E}$ is relatively quasiconvex in $\widetilde{N}$. 
Let $[a, b] \subset \widetilde{M}$ be a geodesic in the intrinsic path metric on $\widetilde{M}$ lying outside a large ball about a fixed reference point $m \in \widetilde{M}$. We want to construct an electroambient $P$-quasigeodesic with respect to $(\widetilde{N}, \mathcal{E})$ lying outside a large ball in $\widetilde{N}$. Towards this, first construct an electro-ambient $P$-quasigeodesic $[a, b]_{q}$ with respect to $(\widetilde{M}, \mathcal{S})$ in $\widetilde{M}$ joining $a, b$ and lying within a $P$ - neighborhood of $[a, b]$ (by Lemma 2.14). This gives us a sequence of points $a=a_{0}, \cdots a_{n}=b$ such that the "odd subpaths" $\left[a_{2 i}, a_{2 i+1}\right]_{q}$ of $[a, b]_{q}$ have interiors disjoint from the elements of $\mathcal{S}$, whereas the "even subpaths" $\left[a_{2 i+1}, a_{2 i+2}\right]_{q}$ of $[a, b]_{q}$ lie entirely within some $\widetilde{S}_{\alpha} \in \mathcal{S}$. Since all these are subpaths of $[a, b]_{q}$, they all lie outside a large ball about $m$. Now replace the even subpaths $\left[a_{2 i+1}, a_{2 i+2}\right]_{q}$ by a geodesic $\overline{a_{2 i+1}, a_{2 i+2}}$ in the corresponding $\widetilde{E}_{\alpha} \in \mathcal{E}$ joining $a_{2 i+1}, a_{2 i+2}$. Since the inclusion of $\widetilde{S}$ into $\widetilde{E}$ has a CT map, it follows that each of the geodesic segments $\overline{a_{2 i+1}, a_{2 i+2}}$ lies outside a (uniformly) large ball about $m$. Concatenate these together by interpolating the odd subpaths $\left[a_{2 i}, a_{2 i+1}\right]_{q}$ of $[a, b]_{q}$. This gives an electro-ambient $P$-quasigeodesic $\overline{a, b}^{q}$ with respect to $(\tilde{N}, \mathcal{E})$ by Remark 2.12 Further, $\overline{a, b}^{q}$ also lies outside a large ball about $m$. Finally, by Lemma 2.14, the hyperbolic geodesic in $\widetilde{N}$ lies in a bounded neighborhood of $\overline{a, b}^{q}$ and hence lies outside a large ball about $m$. Lemma 2.16 now furnishes the required CT map. We now proceed with the general case.

Theorem 3.1. Let $Y, X, \mathcal{H}, \mathcal{J}, \mathcal{B}=\left\{B_{\alpha}\right\}, \mathcal{F}=\left\{F_{\alpha}\right\}, \mathcal{F}_{\alpha}=\left\{F_{\alpha \beta}\right\}$ be as in Definition 2.10 and $i: Y \rightarrow X$ be an ends-inclusion of spaces. Then the ends inclusion $i: Y \rightarrow X$ extends to a Cannon-Thurston map if the inclusions $i_{\alpha}: B_{\alpha} \rightarrow F_{\alpha}$ extend uniformly to Cannon-Thurston maps for all $\alpha$.

Proof. Fix a base point $y \in Y$ and consider a large enough ball $U_{N}(y) \subset Y$. Let $\hat{\eta} \subset \widehat{Y}=\mathcal{E}(Y, \mathcal{H})$ be an electric geodesic segment, starting and ending outside elements of $\mathcal{H}$. Let $\eta^{b}$ denote the bounded part of $\hat{\eta}$ with respect to $(Y, \mathcal{H})$, and assume that it lies outside $U_{N}(y) \subset Y$, i.e $\eta^{b} \cap U_{N}(y)=\varnothing$.

Let $\mathcal{B}_{0}=\left\{B_{\nu} \in \mathcal{B}: \overline{\eta^{b}} \cap B_{\nu} \neq \emptyset\right.$ and $\left.U_{N}(y) \cap B_{\nu} \neq \emptyset\right\}$, where $\overline{\eta^{b}}$ denotes the closure of $\eta^{b}$.

For each $B_{\nu} \in \mathcal{B}_{0}$, let $\eta^{b}(\nu)=\eta^{b} \cap B_{\nu}$. Then $\eta^{b}(\nu)$ lies outside $U_{N}(y) \cap B_{\nu}$. Let $y_{\nu}$ be the nearest point projection of $y$ on $B_{\nu}$ in the metric $d_{Y}$ of $Y$. Since $F_{\nu} \cap Y=B_{\nu}$ it follows that $y_{\nu}$ is also (up to bounded error) a nearest point projection of $y$ on $F_{\nu}$ in the metric $d_{X}$ on $X$. Then $y_{\nu} \in U_{N}(y) \cap B_{\nu}$. Let $d_{Y}\left(y, y_{\nu}\right)=R_{\nu}$. Consider the ball $U_{\left(N-R_{\nu}\right)}\left(y_{\nu}\right)$, of radius $N-R_{\nu}$ about $y_{\nu}$ in $Y$. $U_{\left(N-R_{\nu}\right)}\left(y_{\nu}\right) \cap B_{\nu}$ is a ball in $B_{\nu}(\subset Y)$ of radius $N-R_{\nu}$ based at $y_{\nu}$. We denote this ball as $U(\nu)$. Then $\eta^{b}(\nu) \subset B_{\nu} \backslash U(\nu)$.

Let $\hat{\rho}$ be the electric geodesic in $\widehat{X}=\mathcal{E}(X, \mathcal{J})$ joining the end points of $\hat{i}(\hat{\eta})$. Since $\widehat{Y}$ is weakly hyperbolic rel. $\mathcal{B}$, it follows that $\widehat{X}$ is weakly hyperbolic rel. $\mathcal{F}$. Let the electro-ambient path representative of $\hat{\rho}$ with respect to $\mathcal{F}$ be $\hat{\rho}_{e a}$. Let $\rho_{e a}^{b}=\hat{\rho}_{e a} \backslash \cup_{J \in \mathcal{J}} J$ be the bounded part of $\hat{\rho}_{e a}$ with respect to $(X, \mathcal{J})$. By Remark 2.12. we may assume that $\rho_{e a}^{b} \backslash \cup \bigcup_{F_{\alpha} \in \mathcal{F}} F_{\alpha}=\eta^{b} \backslash \cup \bigcup_{B_{\alpha} \in \mathcal{B}} B_{\alpha}$, i.e. $\rho_{e a}^{b}$ and $\eta^{b}$ coincide outside $\mathcal{F}$.

As per hypothesis, CT maps exist uniformly for each $B_{\nu} \hookrightarrow F_{\nu}$. By Lemma 2.21, there exists a function $M(N) \rightarrow \infty$ as $N \rightarrow \infty$ such that $\rho_{e a}^{b}(\nu)$ lies outside $U_{M\left(N-R_{\nu}\right)}\left(y_{\nu}\right), \forall \nu$. It is worth noting that the function $M(N)$ is independent of $\nu$ by definition of uniformity. 
Since $Y$ is properly embedded in $X$, it follows that there exists a function $M_{1}(N) \rightarrow \infty$ as $N \rightarrow \infty$ such that if $x, y \in Y$ and $d_{Y}(x, y) \geq N$ then $d_{X}(i(x), i(y)) \geq$ $M_{1}(N)$. It follows immediately that $\rho_{e a}^{b} \backslash \cup \bigcup_{F_{\alpha} \in \mathcal{F}} F_{\alpha}$ lies outside $U_{M_{1}(N)}^{X}(i(y))$.

Hence $\rho_{e a}^{b}(\nu)$ lies outside $U_{M_{1}\left(R_{\nu}\right)+M\left(N-R_{\nu}\right)}^{X}(i(y))$ - a ball of radius $M_{1}\left(R_{\nu}\right)+$ $M\left(N-R_{\nu}\right)$ in $X$, i.e.

$$
d_{X}\left(\left(\rho_{e a}^{b}(\nu), i(y)\right) \geq M_{1}\left(R_{\nu}\right)+M\left(N-R_{\nu}\right)\right.
$$

for all $\nu$.

Let $M_{2}(N)=\inf _{\nu}\left(M_{1}\left(R_{\nu}\right)+M\left(N-R_{\nu}\right)\right)$, and $M_{3}(N)=\min \left(M_{1}(N), M_{2}(N)\right.$, which is again a proper function of $N$, i.e. $M_{3}(N) \rightarrow \infty$ as $N \rightarrow \infty$. This proves that $\eta^{b}$ and $\rho_{e a}^{b}$ satisfy the criteria of Lemma 2.21.

Hence, the theorem follows.

An important fact we used in the above proof is that $\rho_{e a}^{b} \backslash \cup \bigcup_{F_{\alpha} \in \mathcal{F}} F_{\alpha}=\eta^{b} \backslash$ $\cup \bigcup_{B_{\alpha} \in \mathcal{B}} B_{\alpha}$, i.e. $\rho_{e a}^{b}$ and $\eta^{b}$ can be chosen to coincide outside $\mathcal{F}$. This followed from Remark 2.12

Now let $\partial i$ denote the Cannon-Thurston map on the boundary $\partial \mathcal{G}(Y, \mathcal{H})$ obtained in Theorem 3.1. We would like to know exactly which points are identified by the CT map $\partial i$. Towards this, we set up some notation.

The inclusions $i_{\alpha}: B_{\alpha} \rightarrow F_{\alpha}$ induce CT maps $\partial i_{\alpha}: \partial \mathcal{G}\left(B_{\alpha}, B_{\alpha \beta}\right) \rightarrow \partial \mathcal{G}\left(F_{\alpha}, F_{\alpha \beta}\right)$ by the hypothesis of Theorem 3.1. Each such map $\partial i_{\alpha}$ induces an equivalence relation $\mathcal{R}_{\alpha}$ on $\partial \mathcal{G}\left(B_{\alpha}, B_{\alpha \beta}\right)$ given by $a \mathcal{R}_{\alpha} b$ if and only if $\partial i_{\alpha}(a)=\partial i_{\alpha}(b)$. Since $\mathcal{G}\left(B_{\alpha}, B_{\alpha \beta}\right)$ is quasiconvex in $\mathcal{G}(Y, \mathcal{H})$ it follows that $\partial \mathcal{G}\left(B_{\alpha}, B_{\alpha \beta}\right)$ embeds homeomorphically in $\partial \mathcal{G}(Y, \mathcal{H})$. Hence $\mathcal{R}_{\alpha}$ induces a natural equivalence relation (also denoted as $\left.\mathcal{R}_{\alpha}\right)$ on $\partial \mathcal{G}(Y, \mathcal{H})$ by identifying points on $\partial \mathcal{G}\left(B_{\alpha}, B_{\alpha \beta}\right)$ with their images under inclusion in $\partial \mathcal{G}(Y, \mathcal{H})$. We shall call the relation $\mathcal{R}_{\alpha}$ on $\partial \mathcal{G}(Y, \mathcal{H})$ the $\mathbf{C T}$ relation induced by $i_{\alpha}$. Let $\mathcal{R}_{t}$ denote the transitive closure of the union $\bigcup_{\alpha} \mathcal{R}_{\alpha}$. Finally, let $\mathcal{R}$ denote the closure of $\mathcal{R}_{t}$ thought of as a subset of $\partial \mathcal{G}(Y, \mathcal{H}) \times \partial \mathcal{G}(Y, \mathcal{H})$ with the product topology. Thus $\mathcal{R}$ is the smallest closed equivalence relation generated by the $\mathcal{R}_{\alpha}$ 's.

As in the discussion preceding Theorem 3.1, we give a quick sketch of what goes on in the special case of a hyperbolic 3-manifold $N$ with incompressible core $M$, one simply degenerate end $E(=N \backslash M)$ and no parabolics. Let $S=E \cap M$ be the single boundary component of $M$. Let $\mathcal{E}=\left\{E_{\alpha}\right\}$ denote the lifts of $E$ to $\widetilde{N}$, $S_{\alpha}=E_{\alpha} \cap \widetilde{M}$, and $\mathcal{S}=\left\{S_{\alpha}\right\}$ be the lifts of $S$ to $\widetilde{M}$. Suppose that $\partial i: \partial \widetilde{M} \rightarrow \partial \widetilde{N}$ denotes the CT map given by Theorem 3.1. Let $\partial i(a)=\partial i(b)$ for $a \neq b \in \partial \widetilde{M}$. Let $\eta \subset \widetilde{M}$ be the bi-infinite geodesic in $\widetilde{M}$ joining $a, b$. Let $a_{n} \rightarrow a$ and $b_{n} \rightarrow b$ be points on $\eta$. Let $\eta_{n}\left(\operatorname{resp} . \rho_{n}\right)$ be the geodesic in $\widetilde{M}(\operatorname{resp} . \widetilde{N})$ joining $a_{n}, b_{n}$. By the converse direction of Lemma 2.14 we can approximate $\rho_{n}$ uniformly by an electro-ambient quasigeodesic $\xi_{n}$ with respect to $(\widetilde{N}, \mathcal{E})$.

We now "reverse-engineer" an electro-ambient quasigeodesic $\left[a_{n}, b_{n}\right]_{q}$ with respect to $(\widetilde{M}, \mathcal{E})$ from $\xi_{n}$ as follows. This step is exactly the opposite of the corresponding step in the sketch before Theorem 3.1. We replace any maximal segment of $\xi_{n}$ lying inside an $E_{\alpha}$ by a geodesic in the corresponding $S_{\alpha} \in \mathcal{S}$ to construct $\left[a_{n}, b_{n}\right]_{q}$. Also, $\left[a_{n}, b_{n}\right]_{q}$ coincides with $\xi_{n}$ outside the $E_{\alpha}$ 's. By Lemma 2.14, $\eta_{n}$ lies in a uniformly bounded neighborhood of $\left[a_{n}, b_{n}\right]_{q}$. Also, since $\left[a_{n}, b_{n}\right]_{q} \backslash \cup \bigcup_{\alpha} S_{\alpha}$ coincides with $\xi_{n} \backslash \cup \bigcup_{\alpha} E_{\alpha}$ and since $\xi_{n}$ converges to $\partial i(a)=\partial i(b)$ as $n \rightarrow \infty$, it follows that $\left[a_{n}, b_{n}\right]_{q}$ converges (in the Hausdorff metric 
on the Gromov compactification $\bar{M})$ to a collection $\cup_{r}\left(c_{r}, d_{r}\right)$ of bi-infinite geodesics, with $\partial i\left(c_{r}\right)=\partial i\left(d_{r}\right)=\partial i(a)=\partial i(b)$ for all $r$. By construction of $\left[a_{n}, b_{n}\right]_{q}$, each $\left(c_{r}, d_{r}\right)$ lies entirely in a single $S_{\alpha}$ and the CT map $\partial i_{\alpha}: S_{\alpha} \rightarrow E_{\alpha}$ identifies $c_{r}, d_{r}$. This shows that the equivalence relation given by the CT map $\partial i: \widetilde{M} \rightarrow \widetilde{N}$ is generated by the equivalence relation given by the CT maps $\partial i_{\alpha}: S_{\alpha} \rightarrow E_{\alpha}$. Corollary 3.2 below generalizes this argument to the relatively hyperbolic setup.

Corollary 3.2. Let $Y, X, \mathcal{H}, \mathcal{J}, \mathcal{B}=\left\{B_{\alpha}\right\}, \mathcal{F}=\left\{F_{\alpha}\right\}, \mathcal{F}_{\alpha}=\left\{F_{\alpha \beta}\right\}$ be as in Definition 2.10 and $i: Y \rightarrow X$ be an ends-inclusion of spaces.

Also, let $\partial i: \partial \mathcal{G}(Y, \mathcal{H}) \rightarrow \partial \mathcal{G}(X, \mathcal{J})$ be the induced Cannon-Thurston map on relative hyperbolic boundaries as in theorem [3.1. Then $\partial i(a)=\partial i(b)$ for $a \neq b \in$ $\partial \mathcal{G}(Y, \mathcal{H})$ if and only if aR $b$ where $\mathcal{R}$ is the smallest closed equivalence relation generated by the $C T$ relations $\mathcal{R}_{\alpha}$ induced by $i_{\alpha}$.

Proof. Let $\mathcal{R}_{Y}$ denote the CT equivalence relation on $\partial Y$ induced by the CT map $\partial Y \rightarrow \partial X$ given by Theorem 3.1. We have to show that $\mathcal{R}=\mathcal{R}_{Y}$.

Since $i_{\alpha}: B_{\alpha} \rightarrow Y$ is a quasi-isometric embedding, it follows that $\mathcal{R}_{\alpha} \subset \mathcal{R}_{Y}$. Hence the transitive closure $\mathcal{R}_{t}$ of the union $\bigcup_{\alpha} \mathcal{R}_{\alpha}$ is also contained in $\mathcal{R}$. Finally, since $\partial i: \partial \mathcal{G}(Y, \mathcal{H}) \rightarrow \partial \mathcal{G}(X, \mathcal{J})$ is continuous, it follows that $\mathcal{R}_{Y}$ is a closed relation. Hence $\mathcal{R} \subset \mathcal{R}_{Y}$.

It remains to show that $\mathcal{R}_{Y} \subset \mathcal{R}$. Suppose that $(a, b) \in \mathcal{R}_{Y}$, i.e. $\partial i(a)=\partial i(b)$ for some $a \neq b \in \partial \mathcal{G}(Y, \mathcal{H})$. Then the geodesic $\eta=(a, b) \subset \mathcal{G}(Y, \mathcal{H})$ satisfies the following:

- If $a_{n}, b_{n} \in(a, b) \subset \mathcal{G}(Y, \mathcal{H})$ are such that $a_{n} \rightarrow a, b_{n} \rightarrow b, \eta_{n}$ is the subsegment of $\eta$ joining $a_{n}, b_{n}$, and $\rho_{n}$ is the geodesic in $\mathcal{G}(X, \mathcal{J})$ joining $a_{n}, b_{n}$, then $d_{\mathcal{G}(X, \mathcal{J})}\left(i(y), \rho_{n}\right) \rightarrow \infty$ as $n \rightarrow \infty$.

By the converse direction of Lemma 2.14 there exists $P \geq 1$ (independent of $\left.a, b, n, a_{n}, b_{n}\right)$ and hyperbolic $P$ - quasigeodesic paths $\xi_{n}$ such that

(1) $\xi_{n}$ is an electro-ambient $P$-quasigeodesic with respect to $(X, \mathcal{F})$ lying within a hyperbolic distance $P$ of $\rho_{n}$.

(2) There exists a sequence of points $a_{n}=a_{n, 0}, a_{n, 1}, \cdots, a_{n, k_{n}}=b_{n}$ on $\xi_{n}$ such that

a) The "odd subpaths" $\overline{a_{n, 2 j}, a_{n, 2 j+1}}$ of $\xi_{n}$ joining $a_{n, 2 j}$ and $a_{n, 2 j+1}$ have interiors disjoint from all $\mathcal{G}\left(F_{\alpha}, F_{\alpha \beta}\right)$.

b) The "even subpaths" $\overline{a_{n, 2 j+1}, a_{n, 2 j+2}}$ of $\xi_{n}$ joining $a_{n, 2 j+1}$ and $a_{n, 2 j+2}$ are entirely contained in some $\mathcal{G}\left(F_{j}, F_{j \beta}\right)$.

By Remark 2.12, the odd subpaths of $\xi_{n}$ joining $a_{n, 2 j}, a_{n, 2 j+1}$ are actually $P$-quasigeodesics in $\mathcal{G}(Y, \mathcal{H})$. Also, since $d_{\mathcal{G}(X, \mathcal{J})}\left(i(y), \rho_{n}\right) \rightarrow \infty$ as $n \rightarrow \infty$, it follows that $d_{\mathcal{G}(Y, \mathcal{H})}\left(y, \overline{a_{n, 2 j}, a_{n, 2 j+1}}\right) \rightarrow \infty$ as $n \rightarrow \infty$. In particular, for all $j$, $d_{\mathcal{G}(Y, \mathcal{H})}\left(y, a_{n, j}\right) \rightarrow \infty$ as $n \rightarrow \infty$.

We shall now reverse the construction used in the proof of Theorem 3.1. Replace the even subpath $\overline{a_{n, 2 j+1}, a_{n, 2 j+2}}$ (of $\left.\xi_{n}\right)$ contained in $\mathcal{G}\left(F_{j}, F_{j \beta}\right)$ by a geodesic $\left[a_{n, 2 j+1}, a_{n, 2 j+2}\right]_{q}$ in the corresponding $\mathcal{G}\left(B_{j}, B_{j \beta}\right)$. Interpolating the odd subpaths of $\xi_{n}$, we obtain an electro-ambient $P$ - quasigeodesic with respect to $\left(\left(\mathcal{G}(Y, \mathcal{H}), \mathcal{G}\left(B_{\alpha},\left\{B_{\alpha \beta}\right\}\right)\right)\right.$. Let $\left[a_{n}, b_{n}\right]_{q}$ denote this electro-ambient $P$ - quasigeodesic.

By Lemma 2.14, the geodesic in $\mathcal{G}(Y, \mathcal{H})$ joining $a_{n}, b_{n}$ lies in a $K(=K(P))-$ neighborhood of $\left[a_{n}, b_{n}\right]_{q}$. Passing to a subsequence if necessary, let $\left[a_{n}, b_{n}\right]_{q}$ converges to $[a, b]_{q}$ in the Hausdorff topology on the Gromov compactification $\overline{\mathcal{G}(Y, \mathcal{H})}$ of $\mathcal{G}(Y, \mathcal{H})$. Let $(a, b)_{q}=[a, b]_{q} \cap \mathcal{G}(Y, \mathcal{H})$. Then the geodesic $\eta$ lies in a $K-$ 
neighborhood of $(a, b)_{q}$. Thus $(a, b)_{q}$ is a countable union of bi-infinite geodesics $\left(c_{r}, d_{r}\right) \subset \mathcal{G}(Y, \mathcal{H})$, such that $\eta$ lies in a $K-$ neighborhood of $\cup_{r}\left(c_{r}, d_{r}\right)$. Here $c_{r}, d_{r} \in \partial \mathcal{G}(Y, \mathcal{H})$. Also, each such $\left(c_{r}, d_{r}\right)$ is a limit of geodesic segments contained in some (sequence of) $\mathcal{G}\left(B_{\alpha},\left\{B_{\alpha \beta}\right\}\right)$. Hence (passing to a further subsequence if necessary) we can assume that each $\left(c_{r}, d_{r}\right)$ is contained in some $\mathcal{G}\left(B_{r},\left\{B_{r \beta}\right\}\right)$.

Again, since each $c_{r}$ or $d_{r}$ is a limit of points $\left(a_{n, r}\right)$ on $\xi_{n}$ and since $d_{\mathcal{G}(X, \mathcal{J})}\left(i(y), \xi_{n}\right)$ $\rightarrow \infty$ as $n \rightarrow \infty$, all the $c_{r}, d_{r}$ get identified with $a, b$ under the CT map $\partial i$.

Further, by the construction of $\left[a_{n}, b_{n}\right]_{q}$ from $\xi_{n}$, it follows that for any $r$, the pair $c_{r}, d_{r}$ get identified with each other under the CT map $\partial i_{r}$ (corresponding to the inclusion of $\mathcal{G}\left(B_{r},\left\{B_{r \beta}\right\}\right)$ in $\left.\mathcal{G}\left(F_{r},\left\{F_{r \beta}\right\}\right)\right)$. Hence $\left(c_{r}, d_{r}\right) \in \mathcal{R}_{r}$, where $\mathcal{R}_{r}$ is the CT-relation induced by $\partial i_{r}$.

Finally, since $\eta$ lies in a $K$ - neighborhood of $\cup_{r}\left(c_{r}, d_{r}\right)$, it follows that the pair $(a, b)$ is contained in the smallest closed relation on $\partial \mathcal{G}(Y, \mathcal{H}) \times \partial \mathcal{G}(Y, \mathcal{H})$ generated by $\mathcal{R}_{r}$, i.e. $(a, b) \in \mathcal{R}$. Hence $\mathcal{R}_{Y} \subset \mathcal{R}$ and the proof is complete.

3.2. Kleinian Groups with no Accidental Parabolics. The first application of Theorem 3.1 is to prove the existence of Cannon-Thurston maps for pared 3manifolds with incompressible boundary and no accidental parabolics. We recall the main Theorem of Mj14a. Let $S$ be a complete finite area hyperbolic surface with fundamental group $H$. Nontrivial elements of $H$ represented by peripheral loops of $S$ are called parabolic elements of $H$. Let $\widetilde{S}$ denote the universal cover of $S$. Note that $\widetilde{S}$ is isometric to $\mathbb{H}^{2}$. Let $\bar{S}=\widetilde{S} \cup S^{1}$ denote the Gromov compactification of $\widetilde{S}$. For $\rho$ a discrete faithful representation of $H$ into $\operatorname{Isom}\left(\mathbb{H}^{3}\right)$ taking parabolics to parabolics, $\Gamma=\rho(H)$ is called a surface Kleinian group. If, in addition, $\rho$ does not send any non-parabolic element of $H$ to a parabolic, then $\Gamma$ is a surface Kleinian group without accidental parabolics. In Theorem 3.3 below, the convex core of $\mathbb{H}^{3} / \Gamma$ will be denoted by $M$ and the union of $\widetilde{M}$ with its limit set will be denoted by $\bar{M}$. We are now ready to recall the main Theorem of $\mathrm{Mj} 14 \mathrm{a}$ :

Theorem 3.3. Mj14a Let $\rho$ be a representation of a surface group $H$ (corresponding to the surface $S$ ) into Isom $\left(\mathbb{H}^{3}\right)$ without accidental parabolics. Let $M$ denote (the convex core of) $\mathbb{H}^{3} / \rho(H)$. Further suppose that $i: S \rightarrow M$, taking parabolics to parabolics, induces a homotopy equivalence. Then the inclusion $\tilde{i}: \widetilde{S} \rightarrow \widetilde{M}$ extends continuously to a map of the compactifications $\bar{i}: \bar{S} \rightarrow \bar{M}$.

Theorem 3.4. Suppose that $N^{h} \in H(M, P)$ is a hyperbolic structure on a pared manifold $(M, P)$ with no accidental parabolics. Further suppose that $N^{h}$ is not a doubly degenerate manifold. Let $M_{g f}$ denotes a geometrically finite hyperbolic structure adapted to $(M, P)$, then the map $i: \widetilde{M_{g f}} \rightarrow \widetilde{N^{h}}$ extends continuously to a map of the compactifications $\bar{i}: \overline{M_{g f}} \rightarrow \overline{N^{h}}$.

Proof. We first show that the lift of each end to $\widetilde{N^{h}}$ is relatively quasiconvex. Suppose not.

Then by Lemma 2.25 a lift $\widetilde{E}$ of an end of $N^{h}$ to $\widetilde{N^{h}}$ is not relatively quasiconvex in $\widetilde{N^{h}}$ if and only if there is a component $F$ of $\delta_{0} M$ such that

(1) $F$ bounds a degenerate end other than $E$, and

(2) $F$ is homotopic into $E$. 
If $F$ is isotopic to a proper subsurface of $\partial E$, then the boundary curves of $F$ necessarily have to be accidental parabolics contadicting the hypothesis.

Else $F$ is isotopic to all of $\partial E$, forcing $N^{h}$ is to be a doubly degenerate manifold and again contadicting the hypothesis.

Hence the map $i: \widetilde{M_{g f}} \rightarrow \widetilde{N^{h}}$ is an ends-inclusion.

The Theorem is now immediate consequence of Theorems 3.1 and 3.3 .

Remark 3.5. For the proof of Theorem 3.4 to work it suffices to assume that each augmented end of $N^{h}$ is relatively quasiconvex. This will be useful in the next subsection when we deal with accidental parabolics.

To state the next theorem describing the point-preimages of the CT map, we set up some notation. Let $N$ be (the convex core of) a hyperbolic structure on a pared manifold $(M, P)$ with relative Scott core $M_{g f}$. Let $\mathcal{E}=\left\{E_{\alpha}\right\}$ denote the lifts of the (relative) ends of $N$ (i.e. the components of $N \backslash M_{g f}$ ). Let $\mathcal{S}_{\alpha}=E_{\alpha} \cap \widetilde{M_{g f}}$. Let $\mathcal{L}_{\alpha}$ denote the lift of the ending lamination (for the end corresponding to $E_{\alpha}$ ) to $\mathcal{S}_{\alpha}$. Each $\mathcal{L}_{\alpha}$ induces an equivalence relation $\mathcal{R}_{\alpha}$ on $\partial \widetilde{M_{g f}}$ as follows:

$a \mathcal{R}_{\alpha} b$ if and only if $a, b$ are ideal end-points of a leaf or complementary ideal polygon of $\mathcal{L}_{\alpha}$. Let $\mathcal{R}$ be the smallest closed equivalence relation (with respect to the product topology on $\partial \widetilde{M_{g f}} \times \partial \widetilde{M_{g f}}$ ) containing all the equivalence relations $\mathcal{R}_{\alpha}$.

In $\mathrm{Mj} 14 \mathrm{~b}$ we also identify the point pre-images of the Cannon-Thurston map.

Theorem 3.6. Mj14b Let $G$ be a simply degenerate surface Kleinian group without accidental parabolics. Then the Cannon-Thurston map $\partial i: \partial \widetilde{S} \rightarrow \partial \widetilde{M}$ from the (relative) hyperbolic boundary of $G$ (which is the same as $\partial \widetilde{S}$ ) to its limit set identifies precisely the end-points of leaves of the ending laminations. More precisely, let $\mathcal{R}$ denote the equivalence relation on $\partial \widetilde{S}$ given by a $\mathcal{R} b$ iff $a, b$ are endpoints of $a$ (lift of a) leaf of the ending lamination or ideal boundary points of a complementary ideal polygon. Then $\partial i(a)=\partial i(b)$ if and only if $a \mathcal{R} b$.

Now, combining Theorem 3.4, Corollary 3.2 and Theorem 3.6 we get:

Theorem 3.7. Suppose that $N^{h} \in H(M, P)$ is a hyperbolic structure on a pared manifold $(M, P)$ such that that $N^{h}$ has no accidental parabolics. Let $M_{g f}$ denotes a geometrically finite hyperbolic structure adapted to $(M, P)$. Let $\partial i: \partial \widetilde{M_{g f}} \rightarrow \partial \widetilde{N^{h}}$ be the Cannon-Thurston map extending $i: \widetilde{M_{g f}} \rightarrow \widetilde{N^{h}}$. Then $\partial i(a)=\partial i(b)$ for $a \neq b$ if and only if $(a, b) \in \mathcal{R}$, where $\mathcal{R}$ is the smallest closed equivalence relation containing the equivalence relations $\mathcal{R}_{\alpha}$.

3.3. Accidental Parabolics. We shall now proceed to remove the restriction on accidental parabolics from Theorem 3.4. The proof proceeds by applying Theorems 3.1 and 3.3 twice successively.

Theorem 3.8. Suppose that $N^{h} \in H(M, P)$ is a hyperbolic structure on a pared manifold $(M, P)$ with incompressible boundary $\partial_{0} M$. Suppose further that $N^{h}$ is not doubly degenerate. Let $M_{g f}$ denotes a geometrically finite hyperbolic structure adapted to $(M, P)$. Then the map $i: \widetilde{M_{g f}} \rightarrow \widetilde{N^{h}}$ extends continuously to the boundary $\bar{i}: \overline{M_{g f}} \rightarrow \overline{N^{h}}$.

Let $\partial i: \partial \widetilde{M_{g f}} \rightarrow \partial \widetilde{N^{h}}$ be the resulting Cannon-Thurston map extending $i$ : $\widetilde{M_{g f}} \rightarrow \widetilde{N^{h}}$. Then $\partial i(a)=\partial i(b)$ for $a \neq b$ if and only if $(a, b) \in \mathcal{R}$, where $\mathcal{R}$ is the 
smallest closed equivalence relation containing the equivalence relations generated by lifts of the ending laminations to $\widetilde{M_{g f}}$.

Proof. First note that by Theorem 3.4 and Remark 3.5, the Theorem follows when each augmented end is relatively quasiconvex. Next, by Lemma 2.25, it follows that an augmented end $E$ of $N^{h}$ is not relatively quasiconvex if and only if there is a component $F$ of $\delta_{0} M$ such that

(1) $F$ bounds a degenerate end other than $E$, and

(2) $F$ is homotopic into $E$.

We construct another hyperbolic structure $W^{h} \in H(M, P)$ as follows:

For each augmented end $E$ of $N^{h}$ that is not relatively quasiconvex, let $F(E, i), i=$ $1, \cdots k_{E}$ be the collection of components of $\delta_{0} M$ satisfying the 2 conditions above. Replace the degenerate end having $F(E, i), i=1, \cdots k_{E}$ as boundary by a geometrically finite end. We repeat this for every augmented end that is not relatively quasiconvex. The resulting hyperbolic structure is denoted by $W^{h}$. We identify $W^{h}$ with its convex core for convenience, i.e. we excise the geometrically finite (flaring) ends.

Each augmented end $E$ of $W^{h}$ is now relatively quasiconvex. By Theorem 3.4 and Remark 3.5. the map $j: \widetilde{M_{g f}} \rightarrow \widetilde{W^{h}}$ extends continuously to the boundary $\bar{j}: \overline{M_{g f}} \rightarrow \overline{W^{h}}$.

Each $F(E, i)$ is parallel to a subsurface of $\partial E$ and hence no other degenerate end can have boundary parallel to a subsurface of $F(E, i)$ unless $N^{h}$ is doubly degenerate (excluded by hypothesis). It follows that the augmented ends bounded by $F(E, i)$ are relatively quasiconvex in $N^{h}$. Hence the inclusion $j_{2}: \widetilde{W^{h}} \rightarrow \widetilde{N^{h}}$ is an ends-inclusion and, by Theorem 3.4 and Remark 3.5 extends continuously to the boundary $\overline{j_{2}}: \overline{W^{h}} \rightarrow \overline{N^{h}}$.

Since $i=j_{2} \circ j$, it follows that the map $i: \widetilde{M_{g f}} \rightarrow \widetilde{N^{h}}$ extends continuously to the boundary $\bar{i}: \overline{M_{g f}} \rightarrow \overline{N^{h}}$.

The last statement follows from (applying twice) the structure of the CannonThurston map given by Theorem 3.7

\section{REFERENCES}

[Ago04] I. Agol. Tameness of hyperbolic 3-manifolds. preprint, arXiv:math.GT/0405568, 2004.

[Bon86] F. Bonahon. Bouts de varietes hyperboliques de dimension 3. Ann. Math. vol.124, pages 71-158, 1986.

[Bow12] B. H. Bowditch. Relatively hyperbolic groups. Internat. J. Algebra and Computation. 22, 1250016, 66pp, 2012.

[Can96] R. D. Canary. A covering theorem for hyperbolic 3 manifolds. Topology 35, pages 751778,1996

[Far98] B. Farb. Relatively hyperbolic groups. Geom. Funct. Anal. 8, pages 810-840, 1998.

[GC06] D. Gabai and D. Calegari. Shrink-wrapping and the Taming of Hyperbolic 3-manifolds. J. Amer. Math. Soc. 19, pages 385-446, 2006.

[Gro85] M. Gromov. Hyperbolic Groups. in Essays in Group Theory, ed. Gersten, MSRI Publ.,vol.8, Springer Verlag, pages 75-263, 1985.

[Kap01] M. Kapovich. Hyperbolic Manifolds and Discrete Groups. Progress in Mathematics, vol. 183, Birkhauser, 2001.

[Kla99] E. Klarreich. Semiconjugacies between Kleinian group actions on the Riemann sphere. Amer. J. Math 121, pages 1031-1078, 1999.

[Mit98] M. Mitra. Cannon-Thurston Maps for Trees of Hyperbolic Metric Spaces. Jour. Diff. Geom.48, pages 135-164, 1998. 
[Mj09] M. Mj. Cannon-Thurston Maps for Pared Manifolds of Bounded Geometry. Geom. Topol. 13, arXiv:math.GT/0503581, pages 189-245, 2009.

[Mj10] M. Mj. Cannon-Thurston Maps for Kleinian Groups. preprint, arXiv:math 1002.0996, 2010.

[Mj14a] M. Mj. Cannon-Thurston Maps for Surface Groups. Ann. of Math., 179(1), pages 1-80, 2014.

[Mj14b] M. Mj. Ending Laminations and Cannon-Thurston Maps, with an appendix by S. Das and M. Mj. Geom. Funct. Anal. 24, pages 297-321, 2014.

[MP11] M. Mj and A. Pal. Relative Hyperbolicity, Trees of Spaces and Cannon-Thurston Maps. Geom. Dedicata 151, pages 59-78, 2011.

[Ota98] J. P. Otal. 'Thurston's hyperbolization of Haken manifolds. 'Surveys in differential geometry, Vol. III Cambridge, MA, Int. Press, Boston, MA, pages 77-194, 1998.

[Thu80] W. P. Thurston. The Geometry and Topology of 3-Manifolds. Princeton University Notes, 1980.

[Thu86a] W. P. Thurston. Hyperbolic Structures on 3-Manifolds,I: Deformations of acylindrical manifolds. Ann. of Math. 124, pages 203-246, 1986.

[Thu86b] W. P. Thurston. Hyperbolic Structures on 3-Manifolds,III: Deformations of 3-manifolds with incompressible boundary. preprint, 1986.

School of Mathematical Sciences, RKM Vivekananda University, Belur Math, WB711202 , INDIA 